\title{
On a theorem of AUSLANDER and LYNDON: A correction
}

\author{
$\mathrm{By}$ \\ HANAA NEUMANN
}

Dr. D. E. CoHes has pointed out to me that the proof of Lemma 1 in my note [1] is false, and apparently the gap left by the mistake can not be closed completely by elementary arguments - 'elementary' in the spirit of that note. More recently I have again made use of just that Lemma $I$ in a similar context (cf. [2], Lemma 2.1). The following lines serve the dual purpose to make clear just what gap is left in the note [1], and to point out that the truth of Lemma 2.1 in the note [2] is not affected.

In [I] we were concerned with the following theorem:

If $R$ is a normal subgroup of a non-abelian free group $F$ such that for some integer $n \geqq 3$ the $n$-th term $F_{n}$ of the lower central series of $F$ is contained in $[R, R]$, then $R=F$.

Lemma 1 served to reduce the proof of the general case to that of a free group of rank two. To this end, we now note first that it suffices to assume that $R$ contains $F^{\prime}=[F, F]$. For otherwise consider $S=R F^{\prime}$. The theorem applied to $S$ gives $S=F$; but as the assumptions make $F / R$ nilpotent, $R F^{\prime}=F$ implies $R=F$.

Now assume the theorem true in every free group of rank two and apply to the given situation a retractive endomorphism $\varepsilon$ that maps $F$ onto a free factor of rank two. One obtains: $R \varepsilon=F \varepsilon$ for every such retraction $\varepsilon$. In the 'proof' in [1] I stated that $R \varepsilon=R \cap F \varepsilon-$ which is false (but true when $R$ is fully invariant in $F$ ); nor is it true that $R \varepsilon=F \varepsilon$ for every such retraction implies $R=F$, as is shown by taking in the free group of countably infinite rank a normal subgroup $R$ such that $F / R$ is the additive group of rationals. We have, however:

Lemma. If $F / R$ is finitely generated or of finite exponent, then $R \varepsilon=F \varepsilon$ for every retractive endomorphism of $F$ onto a free factor of rank two implies $R=F$.

For in this case $F / R$ is a direct product of cyclic groups and so, with a suitable choice of the free generators $e_{i}$ of $F, R$ may be assumed to be generated by powers of the $e_{i}$ modulo $F^{\prime}$; that is $R=\operatorname{gp}\left(F^{\prime}, e_{i}^{\alpha_{i}}\right)$ where the $\alpha_{i}$ are non-negative integers. Now use just those retractions mapping $F$ onto the free factor generated by $e_{i}$ and $e_{j}$ for all pairs $i, j$ and $R=F$ follows.

This deals with the case of free groups of finite rank; I do not know how the given fact $F_{n} \leqq[R, R]$ may be used in an elementary manner to exclude awkward cases as the one mentioned above when $F$ has infinite rank.

We turn to Lemma 2.1 of the note [2]. Denoting by $G_{p}$ the least normal subgroup of the group $G$ such that $G / G_{p}$ is abelian of exponent $p$, the lemma states: 
If $R$ is a normal subgroup of the non-abelian free group $F$ such that $F_{p} \leqq R$ and $\left[F_{p}, F\right] \leqq R_{p}$, then either $R=F$ or $p=2$ and $R$ is of index two in $F$.

Again one deduces first from the validity of the theorem in free groups of rank two that for every retraction $\varepsilon$ on a free factor of rank two one has either $R \varepsilon=F \varepsilon$ or $p=2$ and $R \varepsilon$ is of index two in $F \varepsilon$. This time the assumptions ensure that $F / R$ is of exponent $p$ and so the Lemma above does in fact permit to conclude that either $R=F$ or $R$ is of index two in $F$.

\section{References}

[1] Handa Neumanx, On a theorem of Auslander and Lyndon. Arch. Math. 13, 1-3 (1962).

[2] Havia Nedrans, Note on a theorem by Gaschütz. J. reine angew. Math. 212, 109-112 (1963).

\section{Eingegangen am 29. 3. 1963}

Anschrift des Autors:

Hanna Neumann

Department of Mathematics, I.A.S.

The Australian National University

Box 4, G.P.O.

Canberra, A.C.T.

Australia 